\title{
Eine Ethik des unzuverlässigen Erzählens. Fred Wanders Erzählung Der siebente Brunnen (1971)
}

\author{
Stephan Pabst
}

Zusammenfassung Fred Wanders Erzählung Der siebente Brunnen ist eine Gegenerzählung zur antifaschistischen Legitimationserzählung Nackt unter Wölfen von Bruno Apitz. Das äußert sich auch in der erzählerischen Form. Wander arbeitet mit einem unzuverlässigen Erzähler. Er motiviert diese Unzuverlässigkeit ethisch und epistemisch mit der Erzählsituation, die der Holocaust historisch hervorgebracht hat. Daran lässt sich auch zeigen, warum - wenigstens bei der Beschreibung literarischer Texte - der Begriff der Unzuverlässigkeit dem des Traumas vorzuziehen ist.

Nach 1989 waren die Erwartungen groß, die Schubladen ostdeutscher Schriftsteller seien mit all den Texten gefüllt, die sie in der DDR nicht veröffentlichen konnten, aber - Dissidenten, die sie sein sollten - geschrieben hatten. Daraus sprach vielleicht die Neugier auf eine, andere` Literatur (vgl. Arnold 1990), sicher aber ein gewisser Überdruss an der bundesdeutschen Gegenwartsliteratur, deren historische Erschöpfung in der Wohlstandsstagnation der 1980er Jahre einige KritikerInnen satt hatten (vgl. Radisch 1998), und die etwas romantische Vorstellung einer Literatur, die unabhängig von ihren Erscheinungsbedingungen einfach entsteht. Die Schubladen aber, in denen man in den 1990er Jahren kramte, erwiesen sich im Wesentlichen als leer.

Trotzdem gab und gibt es in Bezug auf die sogenannte DDR-Literatur Entdeckungen zu machen. Es sind Bücher, die zwar mit dem Segen der Hauptverwaltung Verlage und Buchhandel erschienen waren, die aber aufgrund fehlender Presse in der DDR und in der BRD - die ja auch einen Anerkennungsfaktor für Bücher aus der DDR darstellte - geringer Auflagenzahl oder anderweitig

\author{
S. Pabst $(\bowtie)$ \\ Germanistisches Institut, \\ Martin-Luther-Universität Halle-Wittenberg, \\ Haale (Saale), Deutschland \\ E-Mail: stephan.pabst@germanistik.uni-halle.de
}


erschwerter Zugänglichkeit im Buchhandel, wenig Aufmerksamkeit erfuhren und die auch nach 1989 den Erwartungen an verbotene, dissidentische Bücher nicht entsprachen.

$\mathrm{Zu}$ diesen Büchern gehört die Erzählung Der siebente Brunnen von Fred Wander, die 1971 in der DDR und 1972 in der BRD erschienen war, aber aus unterschiedlichen Gründen hier wie dort ein Geheimtipp blieb. In der DDR wurde die Erzählung mehrfach ${ }^{1}$ und teilweise prominent, u. a. von Christa Wolf (1972) und Irmtraud Morgner (1973) besprochen und ihr Autor mit dem HeinrichMann-Preis ausgezeichnet. Die Laudatio bei der Preisverleihung in der Berliner Akademie der Künste hielt Eduard Claudius. ${ }^{2}$ Trotzdem erreichte sie, auch weil die Auflage mit 5000 Exemplaren klein gehalten wurde, kein größeres Publikum. In Alfred Wellms Roman Morisco heißt es:

Wir sprachen stundenlang über Jaschko [sic!] und seine Brüder, die mit den anderen Kindern in der Baracke eines Todeslagers lebten. [...] Wie kommt es, sagte Anna, daß man über manche Bücher gar nichts hört, man nichts in den Zeitungen liest, während andere Bücher, die unglaubwürdig sind und uns gar nicht berühren, weil die Menschen in ihnen keine Wahrheit haben, gelobt werden und viele Rezensionen haben? (Wellm 1987, S. 111 f.)

Erst in den 1990er Jahren, im Kontext der steigenden Aufmerksamkeit für den Holocaust und die Literatur, die er hervorgebracht hatte, wird die publizistische Arbeit an Wander dichter. 2005 greift der Wallstein-Verlag mit der Neuauflage von Der siebente Brunnen als „Roman“ diese Entwicklung auf. Wanders Text wird nun in eine Reihe mit den Texten Levis, Kertész’ und Semprúns gestellt (vgl. Böttiger 2005; Deiss 2005; Meller 2005).

\section{Ein unzuverlässiger Erzähler ohne unzuverlässige Erzählung}

Die Marginalisierung der Erzählung Der siebente Brunnen in der DDR hing mit ihrem Gegenstand zusammen. Wander, der als Jude von den Nationalsozialisten verschleppt worden war, kehrte nach dem Ende seiner Gefangenschaft zunächst nach Wien zurück und übersiedelte 1958 in die DDR. In seinem Text erzählt er eine Deportationsgeschichte durch unterschiedliche nationalsozialistische Lager aus einer jüdischen Perspektive. Sie endet im Konzentrationslager Buchenwald, dem für die antifaschistische Legitimationserzählung der DDR zentrale Bedeutung

\footnotetext{
${ }^{1}$ Rezensionen erschienen im Sonntag, in der $n d l$, in Sinn und Form, in der Weltbühne, im ND und in der Berliner Zeitung.

${ }^{2}$ Vgl. Sonntag Nr. 16, 16. April 1972, 8. Die Lizenzausgabe im Frankfurter Röderberg-Verlag blieb nahezu unbeachtet. Für die Luchterhand-Ausgabe von 1985 weist das KLG lediglich eine Rezension in der FAZ (vgl. FAZ 17.04.1985) und ansonsten lediglich eine Besprechung in den Salzburger Nachrichten (13.07.1985) nach.
} 
zukam. Diese Bedeutung manifestierte sich 1958 in der Eröffnung der Nationalen Mahn- und Gedenkstätte Buchenwald und fand erzählerischen Ausdruck in Bruno Apitz' im selben Jahr erschienenem Roman Nackt unter Wölfen, der in der DDR lange als historisch verbindliche Lagererzählung betrachtet wurde. Der Roman über die Rettung eines jüdischen Kindes und die Befreiung des Lagers durch kommunistische Häftlinge war praktisch im Moment seines Erscheinens zur hegemonialen Erzählung geronnen. ${ }^{3}$

Dem Bild des Lagers, das Apitz' Roman festschrieb, entsprach Wanders Darstellung nicht. Eine ganze Reihe von erzählerischen Entscheidungen Wanders können geradezu als Einwand gegen Apitz gelesen werden. ${ }^{4}$ Apitz erzählt von politischen Häftlingen und siedelt die Handlung im sogenannten Großen Lager an, in dem sich die Haftbedingungen von denen des Kleinen Lagers stark unterschieden. Gerade in der Endphase des Lagers, von der beide Texte erzählen, waren hier vor allem jüdische Häftlinge, die aus den aufgelösten Lagern in Polen nach Deutschland deportiert worden waren, unter katastrophalen Umständen untergebracht. ${ }^{5}$ Jüdische Häftlinge tauchen bei Apitz nur in der Gestalt des $\mathrm{zu}$ rettenden Kindes auf und als anonyme, morbide Arabeske am Rand des politischen Kampfes. ${ }^{6}$ Die Erzählung endet mit der Rettung des Kindes und der triumphalen ,Selbstbefreiung' des Lagers durch die Häftlinge unter Führung des Internationalen Lagerkomitees. Wander entwirft schon topographisch eine Gegenerzählung, indem er sie im Kleinen Lager und damit auch unter den jüdischen Gefangenen ansiedelt. Er erzählt vom Überleben mehrerer Kinder, was der Geschichte des Lagers besser entspricht, ${ }^{7}$ die ihr Überleben eher sich selbst und ihrer Skrupellosigkeit verdanken als den Anstrengungen politischer Kollektive. Die Befreiung des Lagers registriert der Erzähler eher am Rand, ungläubig und im Zustand der Agonie, ohne sie genau einordnen zu können.

Die Differenzen zwischen beiden Büchern zeigen sich auch auf der Ebene der narrativen Mittel, die sie wählen, und des Geltungsanspruchs, den sie damit erheben. Apitz lässt seinen Roman von einer nullfokalisierten, heterodiegetischen Instanz erzählen, die in den Kopf jedes Häftlings und jedes SS-Mannes und in

\footnotetext{
${ }^{3}$ Vgl. zur Rezeptionsgeschichte des Romans Hähnel und Lemke (1987); Stein (2000); Niven (2009, S. 135-138). Allerdings hat die jüngere Forschung zu Apitz auch gezeigt, dass der Roman nicht einfach aus der ideologischen Funktion erklärt werden kann, die er in der DDR hatte. Vgl. Pabst (2021) und Bogdal (2021).

${ }^{4} \mathrm{Zu}$ Wander als Gegenbuch zu Apitz vgl. Schmidt (2006, S. 408, 412 f.).

${ }^{5}$ Einen detaillierten Einblick gibt Greiser (2001).

${ }^{6}$ Dass Apitz' Roman den Holocaust ,infantilisiere“, wie Ruth Klüger (1992, S. 75) meint, kann eben auch so gelesen werden, dass er den Holocaust sentimental in der Gestalt des geretteten Kindes darstelle. Vgl. Oelke (2008) und Zur Nieden (1997).

${ }^{7}$ Insgesamt überlebten 900 Kinder und Jugendliche das Lager Buchenwald. 1945 war auf Betreiben von Häftlingen eine eigene Baracke - Baracke 66 - für sie eingerichtet worden. Vgl. https://www.buchenwald.de/400/ (abgerufen am 19. 02. 2019). Auf diese Baracke bezieht sich die Befreiungsepisode der Erzählung von Wander.
} 
jeden Winkel des Lagers zu blicken vermag. Er wählt also eine Erzählinstanz, die unzuverlässiges Erzählen sehr unwahrscheinlich macht. Zwar wird die Unzuverlässigkeit impersonaler Erzählinstanzen ${ }^{8}$ wie diejenige in Apitz' Roman heute nicht mehr kategorisch ausgeschlossen, aber es ist doch immerhin umstritten, ob diese Möglichkeit für derartige Instanzen überhaupt besteht (vgl. Ohme 2015, S. 131 f.). ${ }^{9}$ Unsicheres Wissen gibt es bei Apitz eigentlich nur aufseiten der SS, während die Häftlinge, wenigstens die führenden des Internationalen Lagerkomitees, ebenso wie die Erzählinstanz über alle Vorgänge im und, sofern es den Vormarsch der alliierten Truppen betrifft, auch außerhalb des Lagers im Bilde sind. Die Identifikation des Lesers mit den Helden des Buches wird jenseits des Konsenses, der in Bezug auf bestimmte ethische Einstellungen bestehen mag, wesentlich dadurch vollzogen, dass der Leser und die kommunistischen Häftlinge Bescheid wissen, während die SS das nicht tut.

Um diesen Anspruch auf Objektivität aufrechtzuerhalten, nimmt Apitz sogar eklatante Widersprüche zu seiner Zeugenschaft in Kauf, die als Autorisierungsstrategie gleichwohl paratextuell mit geführt wird. An zwei Details lässt sich das auf eine besonders dramatische Weise beobachten. Um nämlich erstens auch die Darstellung des Bewusstseins der SS-Leute durch Zeugenschaft zu rechtfertigen, muss sich Apitz, der vormalige KZ-Häftling, mit den SS-Leuten seines Romans identifizieren: „Ich war auch der Reinebothe, ich war auch der Kluttig“ (zit. n. Sauter 1982, S. 148). Diese v. a. im kultur- und erinnerungspolitischen Kontext der DDR kuriose Aussage wird nur fabriziert, um - wie paradox auch immer - auf Zeugenschaft bestehen zu können. Natürlich erfüllt der Satz auch einen populären, wenngleich apokryph überlieferten Topos der realistischen Romanliteratur. Schon Flaubert wollte Madame Bovary gewesen sein. ${ }^{10}$ Was Apitz allerdings zeigt, indem er Flauberts Satz aufnimmt, ist das Spannungsverhältnis von Zeugenschaft und realistischer Erzählung. Zweitens gehörte Apitz zu einer Gruppe von 47 Funktionshäftlingen des Lagers Buchenwald, die als besonders exponierte Zeugen in den letzten Tagen ermordet werden sollten. Weil die Häftlinge Kenntnis von diesem Plan hatten, konnten sich alle 47 verstecken und das Lager so überleben. Apitz selbst überlebte in einem Kanalschacht (vgl. Hantke 2018, S. 116). Die Geschichte dieses Verstecks nimmt er zwar in seinen Roman auf, ordnet sie aber dem stellvertretenden Lagerältesten Pröll zu, während seine Erzählstimme in keiner Weise von dieser Einschränkung seiner Zeugenschaft, die ja notwendig mit

\footnotetext{
${ }^{8}$ Hier folge ich der Begriffsverwendung Marie-Laure Ryans (1981). Der impersonale Erzähler bezeichnet nicht das Gegenteil dessen, was bei Stanzel der personale Erzähler ist, er bezeichnet also keine Erzählperspektive, sondern die fehlende individuelle Kennzeichnung des Erzählers.

${ }^{9}$ Obwohl Ohme und Lang (2018, S. 64-69) zeigen, dass es auch im Fall heterodiegetischer Erzählungen Formen der Unzuverlässigkeit gibt und dass die Behauptung, das eine schließe das andere aus, wesentlich aus den Implikationen des anachronistischen Begriffs ,auktorial/allwissend" resultiert, sind sie doch seltener und strittiger als bei anderen Erzähltypen.

${ }^{10}$ Der Ausspruch Flauberts ist apokryph überliefert (vgl. Porter 2001, S. 7, 40), spielt aber in der Begründungsgeschichte realistischer Literatur eine wichtige Rolle.
} 
seinem Versteck einhergeht, und v. a. die in der Folge umstrittene Darstellung der Befreiung des Lagers betroffen hätte, berührt wird.

Wanders Erzählung hingegen arbeitet mit einer ganz anderen Erzählinstanz. Sie stellt sich sowohl grammatisch als auch epistemologisch auf den Standpunkt des Zeugen. Sie sagt von Anfang an „Ich“, wenngleich dieses Ich anonym bleibt. Der Ich-Erzähler kann nicht mit Wander identifiziert werden. ${ }^{11}$ Wander selbst hat gelegentlich Anstoß an der ,Verwechslung“ des „Autor[s] mit dem Erzähler“12 genommen. Zwar teilt er mit seinem Erzähler die Stationen der Deportation: Drancy, Perpignan, Beuthen, Hirschberg, Buchenwald, Crawinkel. Die Anonymität des Ich-Erzählers macht die Identifikation mit Wander möglich. Und die Gattungsbezeichnung Erzählung lässt im Gegensatz zum ,Roman“ die Möglichkeit der faktualen Erzählung wenigstens in einem gewissen Umfang offen. Indes ist es textlogisch so, dass durch die Anonymisierung des Ich-Erzählers der , autobiographische Pakt‘ (Lejeune) mit dem Leser nur unvollständig geschlossen wird, da sich autobiographische Texte durch die Identität des Autors, des Erzählers und des Protagonisten der Erzählung auszeichnen. Wenigstens die Identität von Autor und Erzähler aber wird offengelassen, indem der Name des Erzählers nicht genannt wird. Es kann sich um den Autor handeln, muss es aber nicht. Durch die (potenzielle) Nicht-Identität von Autor und Erzähler verschafft sich der Text auch den Spielraum der Fiktion. Das Ich, das in der Konvention des autobiographischen Textes auf ein bestimmtes reales Subjekt verweist, geht dann also potenziell über die Erfahrungen dieses Subjekts hinaus und kündigt damit den Anspruch streng faktualen Erzählens auf.

Weil Ich-Erzähler den subjektiven Beschränkungen des Wissens und der Wahrnehmung unterliegen, stehen sie natürlich fast zwangsläufig im Verdacht der Unzuverlässigkeit (vgl. Nünning 1998, S. 9; Ohme 2015, S. 130), wenngleich die bloße Tatsache subjektiver Beschränktheit noch keinen unzuverlässigen Erzähler macht. Als unzuverlässig können solche Erzähler nur dann betrachtet werden, wenn der Text den Leser begründet daran zweifeln lässt, dass der Erzähler

\footnotetext{
${ }^{11}$ Jörg Thunecke (1999) hält Wanders Text für „,semiautobiographical“. Schmidt (2006, S. 95) spricht von der „Zeugnisabsicht“, die sich durch den narrativen Modus Wanders artikuliere, was auf die autodiegetische Form der Erzählung bezogen werden kann, ohne dass das einen „dokumentarischen Anspruch“ (Schmidt 2006, S. 95) impliziere, den der Text damit erhöbe. Debazi (2008, S. 101) merkt an, dass der eine mit dem anderen nicht identifiziert werden könne, gibt als Grund aber die falsche Gattungsbezeichnung „Roman“ an, die ja eine Erfindung des Wallstein-Verlags ist. Erzähllogisch liegt der Grund in der partiellen Nicht-Identität zwischen Erzähler und Autor. Mc Glothlin (2005, S. 100) erkennt die Differenz, hält sie aber aufgrund der paratextuellen Hinweise auf Wanders Deportationsgeschichte eher für ein Problem, weil sie dazu führen könnte, dass man den Text nicht ausschließlich als autobiographischen Text liest. Dass das aber für ein Problem gehalten wird, liegt an einer in der Holocaust-Literaturforschung grassierenden Ideologie der Zeugenschaft, die sich eine andere Pragmatik der HolocaustLiteratur jenseits oder neben der Zeugenschaft nicht vorzustellen vermag.

${ }^{12}$ Fred Wander an E. Görner, 27. Januar 2003, (c) Susanne Wander, Wien.
} 
seinen Gegenstand im Rahmen der in der erzählten Welt gültigen epistemischen und ethischen Regeln wiedergibt. Zweifel an der Zuverlässigkeit des Erzählers können durch logische Widersprüche, falsche oder unvollständige Informationen des Erzählers und/oder durch die Charakterisierung des Erzählers hervorgerufen werden. Bei Wander ist Letzteres der Fall. Sein anonymer Ich-Erzähler charakterisiert sich gleich im ersten Kapitel selbst, indem er dem Leser einen anderen Erzähler - Mendel Teichmann - vorstellt. Teichmann ist kein Schriftsteller, sondern ein Erzähler, der die Erzählung als Form der sozialen, mündlichen Unterhaltung beherrscht und der mit dieser Fähigkeit offensichtlich großes Ansehen unter seinen Mithäftlingen genießt. Von diesem Teichmann möchte der Erzähler das Erzählen lernen. Tatsächlich weist ihn Teichmann in diese Kunst ein. Er erzählt ihm die Geschichte eines jungen Mannes, der seinerseits von ihm - Teichmann - das Erzählen habe lernen wollen und sein erzählerisches Unvermögen damit begründet, dass er noch nichts erlebt habe. Er wohne schließlich sein Leben lang in demselben, schäbigen Haus. Just aus diesem Hinweis entwickelt Mendel Teichmann auf den folgenden Seiten eine Erzählung über das Haus, in der er detailliert und ausgesprochen plastisch auf dessen Bewohner und deren Verhältnis zueinander eingeht. Dass er selbst das Haus nie gesehen hat, spielt dabei überhaupt keine Rolle:

Was ist nun mit dem Haus, sage ich verwirrt und bemüht, das Schweigen zu brechen. Das alles haben Sie dort gesehen, in jenem Haus, in dem dieses Bürschlein wohnte? Mendel schaute mich erschrocken an: also Du hast nichts verstanden. Ich rede und rede und du verstehst mich nicht. Ich war nicht draußen, wo er wirklich wohnte. Ist es denn so wichtig, dieses Haus, jenes Haus ... (Wander 1971, S. 13) ${ }^{13}$

Die Passage hat im Text ganz unterschiedliche, auch dramaturgische Funktionen, da ihre fast spielerische Harmlosigkeit die außerordentlich brutale und empathieintensive folgende Passage um den Tod Jossls, eines jungen polnischen Juden, scharf konturiert. Eine ihrer Funktionen besteht darin, den Erzähler zu charakterisieren, sowohl den Erzähler Mendel Teichmann, als auch den anonymen Ich-Erzähler, der nach Mendels Tod an dessen Stelle treten wird. ${ }^{14}$ Es handelt sich scheinbar um einen Erzähler, dem nicht recht zu trauen ist, weil er die Frage, ob seine Erzählung der Wahrheit entspricht oder nicht, für unwichtig hält. Er erfüllt also ein wichtiges Merkmal dessen, was man als unzuverlässigen Erzähler bezeichnet.

\footnotetext{
${ }^{13}$ Ich zitiere Wanders Erzählung nach der Erstausgabe im Folgenden mit der Seitenangabe in Klammern im Text.

${ }^{14}$ Die programmatische Bedeutung der Figur erschließt sich aus der Stellung und der Metareflexivität der Erzählung. Sie stand Wander schon am Beginn seiner Arbeit an der Erzählung vor Augen. An Günter Caspar, der zu diesem Zeitpunkt Leiter des Lektorats für Gegenwartsliteratur war, schreibt er am 25.03.1968: „Die erste Geschichte ,Wie man eine Geschichte erzählt‘ gilt als eine Art Vorwort, und die darin dominierende Gestalt, der jüdische Dichter Mendel Teichmann, wird zur Leitfigur aller Erzählungen“" (Faber und Wurm 1994, S. 277).
} 
Typologisch handelt es sich um einen offen unzuverlässigen Erzähler (vgl. Köppe und Kindt 2014, S. 245-250), ${ }^{15}$ also weder um einen Erzähler, der aufgrund seiner intellektuellen Beschränktheit als Wahnsinniger, Säufer oder Kind seine Unzuverlässigkeit gar nicht offenzulegen vermag, noch um einen Erzähler, der seine Unzuverlässigkeit intentional nicht offenlegt, weil er den Adressaten seiner Rede täuschen will. Natürlich enthält der Text tatsächlich Erfindungen. Die partielle Fiktivität Mendel Teichmanns - und die Funktionalität der Figur im Text hätte auch kaum etwas anderes vermuten lassen - hat Wander selbst eingeräumt. ${ }^{16}$ Allerdings stellt sich die Frage nach der Unzuverlässigkeit normalerweise ohnehin nur hinsichtlich der textimmanenten Darstellung von Wirklichkeit und nicht hinsichtlich dessen, was textextern der Fall gewesen sein mag oder nicht. Deshalb irritiert am Bekenntnis des Erzählers, dass wir in seinem Text kaum Spuren mimetischer Unzuverlässigkeit ${ }^{17}$ finden, die darauf hinweisen könnten, dass sich die ,Wirklichkeit‘ gegen ihre Erzählung bemerkbar machte. Der Text bietet also keine alternative Wirklichkeit zu jener an, die uns vom Erzähler erzählt wird. Der Erzähler markiert seine Unzuverlässigkeit, ohne uns textimmanent Anlass zu der Vermutung zu geben, dass er tatsächlich unzuverlässig erzählt. Da, wo er die Lizenz zur Erfindung tatsächlich in Anspruch nimmt, wie im Fall der Hinrichtung Tadeusz Molls, wird das im Text noch einmal ausdrücklich vermerkt. Was Tadeusz Moll in der letzten Nacht vor seiner Hinrichtung durch den Kopf geht und worüber er mit den anderen Verurteilten gesprochen hat, kann der Erzähler nicht wissen, und von denen, die bei ihm sind, kann er es, da sie mit Moll sterben, auch nicht erfahren. Auf die Frage nach seinen Gedanken gibt es also nur eine ,fiktive Antwort" (119).

Warum aber stellt der Erzähler sein gesamtes Erzählen unter den Vorbehalt der Unzuverlässigkeit, wenn er dann gar nicht unzuverlässig erzählt und die Erfindung da, wo sie aus epistemologischen Gründen ohnehin unvermeidbar ist, eigens ausweist? Es scheint in der Sache der Holocaust-Literatur zu liegen, dass sich diese Frage nicht allein mit Blick auf den Erzähler beantworten lässt. Während sich die Frage der Unzuverlässigkeit normalerweise nur in Bezug auf die fiktive Welt und das Verhältnis des Erzählers zu ihr stellt, ist diese Literatur eine, die ihrem Gegenstand gegenüber immer in gewisser Weise rechtfertigungsbedürftig bleibt und von der Mehrzahl der Leser auch so gelesen wird (vgl. Langer 1995, S. 75-88).

\footnotetext{
${ }^{15}$ Der Begriff des offen unzuverlässigen Erzählens beschränkt sich hier allerdings auf das Bekenntnis des Erzählers zu seiner Unzuverlässigkeit und meint insofern etwas weniger als Kindt/Köppe, die von offen unzuverlässigem Erzählen immer dann sprechen, wenn Texte „in offensichtlicher falscher Weise Angaben über fiktive Tatsachen“ (Faber und Wurm 1994, S. 246) machen. Dass diese Offensichtlichkeit und insofern die Offenheit der Unzuverlässigkeit ausgesprochen strittig sein kann, konzedieren sie selbst (Faber und Wurm 1994, S. 250). Vgl. auch Aumüller (2018).

${ }^{16}$ Zwar habe es eine Person mit den Eigenschaften Teichmanns gegeben, aber an den Namen könne er - Wander - sich ebenso wenig erinnern wie an den Inhalt seiner Rede. Vgl. Trampe (1997, S. 13).

${ }^{17}$ Zum Begriff der mimetischen Unzuverlässigkeit vgl. Kindt (2008, S. 46-52).
} 
In der Regel schließen Leser selbst dann, wenn ein Text über den Holocaust als Erzählung oder als Roman ausgewiesen wird, also Gattungsbezeichnungen führt, die als Fiktionssignal gelesen werden können, nur einen eingeschränkten Fiktionsvertrag oder einen Fiktionsvertrag unter Vorbehalt. Wir wissen, dass der Autor weiß, dass er nicht vollständig von einem gewissen Rechtfertigungsdruck gegenüber der historischen Wirklichkeit des Ereignisses entbunden ist. Das gilt umso mehr, als viele dieser Autoren wie Imre Kertész, H. G. Adler oder Elie Wiesel auch als Zeugen auftreten und sich ihre Autorimago insofern von derjenigen anderer Autoren unterscheidet (wenngleich man sich heute natürlich Gedanken darüber machen könnte, inwiefern das Paradigma der Zeugenschaft Autorschaft prinzipiell verändert hat). Es gehört also zu den spezifischen Rezeptionsregeln der Holocaust-Literatur, dass das Urteil über die Zuverlässigkeit des Erzählers textexternes Wissen einbeziehen kann. In Bezug auf dieses Wissen mag es durchaus einen Widerspruch zwischen Erzählung und Wirklichkeit geben, der dem Autor als Erzähler zur Last gelegt werden könnte und der deshalb durch die Selbstanzeige der Fiktion abgefedert werden muss.

Das gilt umso mehr, als Wanders Text noch in einer Zeit der Zeugenkonkurrenz entsteht. D. h., es gibt nicht nur Leser mit einem sekundären Wissen über die Lager, sondern auch solche, die glaubwürdig behaupten können, ein primäres Wissen zu haben. Das ist eine Rezeptionssituation, die sich heute stark verändert hat, da es unter den Lesern kaum noch Zeugen gibt. Für Wander aber galt sie in der DDR umso mehr, als sein Text unter dem Druck der antifaschistischen Erinnerungshegemonie stand, der sich auf die Wahrnehmung, ja selbst auf die Publikation der Bücher in bedeutendem Maß auswirkte. So war es etwa in den 1950er Jahren ehemaligen kommunistischen Buchenwaldhäftlingen möglich, die Erinnerungen des Juden Rolf Weinstock derart zu diskreditieren, dass das schon gedruckte Buch vom Verlag wieder zurückgezogen wurde. Der Angriff auf das Buch galt der Darstellung des antifaschistischen Buchenwald-Gedenkens, der Befreiung des Lagers und der Bedeutung des illegalen Lagerwiderstands und er wurde durch einen ausgesprochen perfiden Angriff auf die Person des Autors lanciert: „Daß einer als ,Mülltonnenadler' sich betätigte, läßt ihn als Autor eines Buchs über Konzentrationslager nicht besonders geeignet erscheinen“, schrieb Stefan Heymann (1945), der bereits in Buchenwald zum Parteiaktiv der KPD gehörte und später als SED-Funktionär, Botschafter der DDR in Ungarn bzw. Polen und Professor eine starke Sprecherposition innehatte. ${ }^{18}$ Anders gesagt: Die spezifische Situation, in die Weinstock als Jude geriet, der gerade in der Endphase des Lagers Buchenwald sehr viel schlechteren Versorgungsbedingungen unterlag als Angehörige anderer Häftlingsgruppen, ${ }^{19}$ machte ihn als Autor unzuverlässig.

\footnotetext{
${ }^{18}$ Eine Darstellung des Falls gibt Simone Barck (2003, S. 48-54).

${ }^{19}$ Über die katastrophale Versorgung im sogenannten Kleinen Lager und die daraus resultierende Sterblichkeit, die deutlich über dem Lagerdurchschnitt lag, vgl. Knigge (2016, S. 144-148). Allein in den ersten drei Monaten des Jahres 1945 starben dort 6000 Menschen. Vgl. Knigge (2016, S. 145).
} 
Weinstock selbst hat auf diesen Angriff reagiert und nicht nur die gegen ihn erhobenen Vorwürfe der verzerrten Darstellung des Lagers bestritten, sondern auch deutlich gemacht, dass die Sprecherposition des vormaligen Funktionshäftlings Heymann (vgl. Niethammer 1994, S. 503 f.), ihrerseits Beschränkungen unterliegt: „Ich gehörte nicht zu jenen, die das Glück hatten, von den Schreibstuben und sonnigen Stellen aus sich vor Kälte und Hunger zu schützen.“20 Heymann, so der Vorwurf, gehörte zu den ,Privilegierten“ des Lagers. Sein Urteil ist nicht einfach dem Lager geschuldet wie es, wirklich“ war, sondern den relativen ,Privilegien', die er in ihm genoss.

Die Angriffe auf Wander werden etwas respektvoller vorgetragen, funktionieren strukturell aber ähnlich als Angriff auf die Zuverlässigkeit des Autor-Erzählers. Eine Gruppe ehemaliger politischer Buchenwald-Häftlinge kritisiert, dass Wander die Lizenz zur Fiktion überstrapaziert habe, wobei sie wiederum an der Darstellung der Endphase des Lagers Anstoß nehmen. Weil der Lagerwiderstand die Kontrolle über das Lager gehabt habe, sei es zu chaotischen Zuständen, wie sie Wander schildere, nicht gekommen:

Es scheint offensichtlich, daß Du erst in den letzten Tagen in einem Massentransport ins Lager kamst. Und es ist zu vermuten, daß die erwähnte Fleckfiebererkrankung Erlebnisse der verschiedenen Konzentrationslager in Deinem Gedächtnis durcheinander gebracht hat. $^{21}$

Also eine klassische Indikation für unzuverlässiges Erzählen: geistige Beschränkung durch Krankheit. Wanders kurze Antwort auf diesen Brief reagiert auf die systematische Verknüpfung von operativer Hoheit im Lager und epistemischer Hoheit im Rückblick auf das Lager, die von seinen Kritikern hergestellt wurde. Und indem er die eine bestreitet, untergräbt er auch die andere:

Wovon reden wir eigentlich? Von einer Wohlfahrtseinrichtung der illegalen KPD oder von einem Konzentrationslager [...]? [...] Wer könnte behaupten, es habe keine von der illegalen Parteiorganisation kontrollierte [sic] Ereignisse gegeben, das ist doch absurd!22

Wander stellt den epistemischen Anspruch des organisierten kommunistischen Erinnerungskollektivs infrage, indem er daran zweifelt, dass der Lagerwiderstand in den letzten Tagen des Lagers Herr der Lage gewesen sei.

Dabei macht die Rezeptionssituation solcher Texte in der DDR vielleicht nur deutlich, was für Texte über den Holocaust auch über die DDR hinaus gilt: Es ist eine inzwischen einigermaßen verbreitete fiktionstheoretische Annahme, dass die Unterscheidung zwischen fiktionalem und faktualem Erzählen immer nur als konventionalisierte „soziale Praxis“ verstanden werden kann (Köppe 2014, S. 35), die sich weder notwendig und ausschließlich aus den Eigenschaften des Textes noch

\footnotetext{
${ }^{20}$ Rolf Weinstock an den VVN-Verlag, 12.12.1950, zit. n. Barck (2003, S. 49).

${ }^{21}$ Der Brief wurde von Benno Biebel, Otto Halle, Herbert Sandberg und Robert Siewert unterzeichnet. Fred Wander Archiv 1067, AdK.

${ }^{22}$ Fred Wander an Benno Biebel, Otto Halle, Herbert Sandberg, Robert Siewert, 23.06.1971, Akademie der Künste, Anna-Seghers-Archiv 1662, ( ) Susanne Wander, Wien.
} 
aus dem ontologischen Status der von ihm erzählten Ereignisse ergibt. Schlechter erforscht ist, wie sich eine solche Praxis eigentlich ausbildet, wohl auch deshalb, weil man bei ihrer Beschreibung meint, „von bestimmten Schlüssen vom Text auf Sachverhalte in der Wirklichkeit absehen“ zu können (Köppe 2014, S. 35). Offenbar ist es nicht einfach so, dass die Praxis des Unterscheidens einmal etabliert und dann immer wieder angewandt wird, vielmehr hängt sie auch von bestimmten Themen und medialen Veränderungen ab, die die Notwendigkeiten und die Möglichkeiten der Unterscheidung herausfordern. In der Geschichte der sogenannten Holocaust-Literatur fällt auf, dass an ihrem Anfang eben mehrheitlich faktuale Texte stehen und die großen Fiktionen des Holocaust zumeist erst später entstehen. Das ist ein Hinweis darauf, dass sich die Praxis des Unterscheidens zwischen fiktionalem und faktualem Erzählen in Bezug auf den Holocaust erst ausbilden muss und nicht einfach so, wie sie in Bezug auf andere Genres bereits existiert, angewandt werden kann. Insofern reagiert Wanders Text auf eine Situation, in der sich der institutionelle Rahmen der Unterscheidung - unter sehr speziellen ideologischen Bedingungen - noch nicht ausgebildet hat. ${ }^{23}$

Dass Wander also mit der Figur Mendel Teichmanns ein Bekenntnis zum unzuverlässigen Erzählen ablegt, obwohl sein Erzähler dann gar nicht unzuverlässig erzählt, hängt wohl auch damit zusammen, dass er mit einem Leser rechnen muss, der von einer Holocaust-Erzählung faktische Wahrheit erwartet und es deshalb für angemessen hält, den Text mit historischer Wahrheit abzugleichen. Daraus resultiert der Verdacht potenzieller Unzuverlässigkeit, der indes textimmanent nicht sichtbar gemacht werden muss, weil sie sich ohnehin nur aus textexternen Wirklichkeitsannahmen ergibt.

\section{Die Epistemologie des Holocaust}

Darüber hinaus gibt es aber durchaus textimmanente Gründe für die Selbstanzeige der Unzuverlässigkeit, Gründe also, die sich zunächst aus dem Verhältnis des Erzählers zu den von ihm erzählten Ereignissen ergeben, ohne dass dazu in irgendeiner Weise auf den Autor Bezug genommen werden müsste. Die Antwort auf die Frage, warum sich ein Erzähler als unzuverlässig markiert, der dann gar nicht unzuverlässig erzählt, fällt da etwas anders aus. Textimmanent scheint es mir zwei Antworten auf sie zu geben. Die eine könnte man als Epistemologie des Holocaust, die andere als Ethik des Erzählens vom Holocaust bezeichnen. Der Gegenstand der Erzählung - die Holocaust-Erfahrung des Erzählers und der mit ihm Deportierten - unterliegt einigen epistemischen Besonderheiten. Die,

\footnotetext{
${ }^{23}$ Durchaus zum Vorteil des Textes, weil es dem Thema angemessener ist, wenn die Grenze unsicher bleibt.
} 
die gestorben sind, können nicht erzählen. Dieser Umstand ist von der philosophischen Holocaust-Essayistik Jean-Francoise Lyotards (1989, S. 17 f.) und Giorgio Agambens (2003, S. 30) nicht nur festgestellt, sondern in teilweise problematischer Weise zur philosophischen Paradoxie der Holocaust-Zeugenschaft ausgebaut und überhöht worden. ${ }^{24}$ Richtig aber ist, dass eben bestimmte Aspekte der Lagererfahrung nicht oder nur unzuverlässig bezeugt werden können. Durch die hohe Sterblichkeit, die Vielsprachigkeit in den Lagern und die sich häufig ändernde Zusammensetzung der Häftlingsgruppen, durch die Unterschiede zwischen verschiedenen Lagerteilen und deren Trennung voneinander und die relative Abgeschiedenheit der Lager von der sie umgebenden Wirklichkeit, befinden sich die Zeugen oft in einer Situation, in der sie die einzige Quelle des Zeugnisses sind und sich ihre Rede nicht mehr überprüfen lässt. Erzähllogisch befinden sie sich in der Situation eines Odysseus' oder eines Ismaels, des IchErzählers in Herman Melvilles Moby Dick, die beide die einzigen Überlebenden einer ansonsten zeugenlosen Katastrophe sind. Primo Levi, für dessen Bericht Ist das ein Mensch? die Odyssee auf unterschiedlichen Ebenen bedeutsam ist, erzählt immer wieder von solchen zeugenlosen Ereignissen: „Ich habe Schlome nie wiedergesehen“ (Levi 1961, S. 31); und so bleibt der seltene Moment der überwältigenden Sympathie mit einem Mithäftling ohne Bestätigung. Von Wanders Tadeusz Moll erfährt man etwa, dass er - bevor er schließlich im Lager Crawinkel hingerichtet wird - in Auschwitz im sogenannten Sonderkommando eingesetzt war, das die Vergasung der Juden vorbereitete und die Leichen anschließend verbrannte. Gerade diese Häftlinge wurden, wohl auch deshalb, weil sie den gesamten Vernichtungsvorgang bezeugen konnten, und aufgrund geplanter oder wirklicher Rebellion gegen die SS, selbst zum allergrößten Teil ermordet (vgl. Piper 1999, S. 213-224; Greif und Levin 2015, S. 34). Der Erzähler begegnet deshalb der im Kontext des Holocaust unwahrscheinlichen Geschichte von der Rettung Molls durch zwei andere Arbeiter aus dem Sonderkommando mit einem gewissen Unglauben. Weil aber Zeugen fehlen, gibt es gar keinen Ansatzpunkt für die Bestätigung oder Widerlegung der Geschichte Molls:

Wer waren die beiden Männer, Bekannte aus Łódź?

Keine Ahnung.

Kamen sie mit auf Transport?

Hab sie nicht gesehen. Glaub', sie sind alle tot. (101)

Die Rettung Molls hat keine Zeugen und das Zeugnis kann auch nicht durch das schwächere Argument der Wahrscheinlichkeit bezeugt werden. Sie ist ,im Grunde unerklärlich.“"(111).

\footnotetext{
${ }^{24}$ In beiden Fällen wird eine Erwartung von vollständiger und widerspruchsfreier Zeugenschaft vorausgesetzt, die ohnehin - und zwar nicht nur hinsichtlich des Holocaust - nie erfüllt sein dürfte.
} 
Solche Hinweise auf das lagerspezifische Nicht-Wissen des Erzählers finden sich mehrfach in Wanders Text. Scheinbar beiläufig wird nach einer Nacht im Waggon festgestellt: „Es starben an diesem Morgen Bertrand Lederer aus Charleroi und Abram Larbaud aus Montpellier, es starb Efraim Bunzel aus Prag und Samuel Wechberger aus Lodz, wer noch in den Waggons vor und hinter uns starb an diesem Morgen erfuhren wir nicht.“ (52) Zentral aber, gerade in der impliziten Kommentierung des Apitz-Romans, ist der Schluss der Erzählung von Wander. Denn geradezu ostentativ weist der Erzähler darauf hin, dass eines der Kernereignisse der Erzählung von Buchenwald in der DDR - die Selbstbefreiung des Lagers durch politische Häftlinge - nicht im Horizont seiner Zeugenschaft liegt: „Im oberen Lager hörte man Schüsse. Sie erzählten sich, die Politischen kämpften mit Waffen, die sie jahrelang vergraben hatten, gegen die SS. Häftlinge mit Handgranaten und Gewehren?“ (139).

Das prinzipielle Bekenntnis des Erzählers zu seiner Unzuverlässigkeit resultiert also in gewisser Weise aus einem bestimmten, durch die Wirklichkeit des Lagersystems und der Deportationen erst erzeugten Nicht-Wissen. „L'horreur y est obscurité, manque absolu de repère“, schreibt Robert Antelme (2007 [1957], S. 11) über Gandersheim, ein Außenlager Buchenwalds, in dem er zwischen 1944 und 1945 inhaftiert war. Fiktive Elemente stellen eine Reaktion auf dieses NichtWissen dar. Man hat es in Wanders Erzählung also mit einem ganz bestimmten unzuverlässigen Erzähler zu tun: Während sich die Feststellung der Unzuverlässigkeit in der Regel auf Erzähler bezieht, die entweder aufgrund ihrer subjektiven Beschränktheit oder aufgrund subjektiver Täuschungsabsichten, in jedem Fall aber aus subjektiven Gründen unzuverlässig erzählen, leitet dieser Erzähler seine Unzuverlässigkeit aus einer spezifischen Epistemologie ab, die aus der Situation resultiert, in der er sich befand und über die er jetzt erzählt. Er gibt seine Unzuverlässigkeit nicht nur zu erkennen, er verweist auch darauf, dass seine Unzuverlässigkeit in der Sache selbst ihren unhintergehbaren Grund hat. Es handelt sich also um einen aufrichtigen, epistemologisch reflektierten und insofern zuverlässigen unzuverlässigen Erzähler, dessen Unzuverlässigkeit zwar auf einen subjektiven Zustand des Nicht-Wissens verweist, der aber aus den Umständen resultiert, von denen er erzählt.

Dass diese Geste der Aufrichtigkeit ihrerseits korrumpiert werden kann, zeigt der Fall Bruno Doesseckers, der als Binjamin Wilkomirski gefälschte Holocaust-Erinnerungen verfasste und sie unter anderem mit der Geste aufrichtiger Unzuverlässigkeit beglaubigte: „Meine frühesten Erinnerungen gleichen einem Trümmerhaufen“ (Wilkomirski 1995, S. 7). Im Grunde ist das ein besonders dramatischer Fall der Einsicht, dass auch offen unzuverlässiges Erzählen mit einer Täuschungsabsicht einhergehen kann (Lang 2018). ${ }^{25}$

\footnotetext{
${ }^{25}$ Wenngleich mir eine Erzählung wie diejenige Wanders Langs These, dass auch offenes unzuverlässiges Erzählen immer mit Täuschungsabsichten zusammenhänge, nicht zu bestätigen scheint.
} 


\section{Die Ethik des unzuverlässigen Erzählens}

Während sich die dem Text immanente, aber über den Text hinaus plausible Epistemologie des holocaustspezifischen Nicht-Wissens eher auf die mimetischen Aspekte der Unzuverlässigkeit bezieht, bezieht sich der zweite Grund der Unzuverlässigkeitsannonce eher auf deren axiologische Aspekte. ${ }^{26}$ Denn zunächst könnte man es ja als ethisches Skandalon empfinden, dass mit einer gewissen Frivolität die Erzählung vom Holocaust als erzählerischer Zusammenhang eingeführt wird, in dem nicht nur aus epistemologischen Gründen über den Wirklichkeitsgehalt des Erzählten keine endgültige Aussage getroffen wird, sondern in dem der Erzähler diese Unentscheidbarkeit sogar noch gut heißt. Was also in Bezug auf Mendel Teichmanns Erzählung über ein Haus in seiner Indifferenz ethisch unproblematisch ist, wird in Bezug auf den Gegenstand, den der Ich-Erzähler hat, sehr wohl zum Problem. Tatsächlich besteht bis heute begründeter Zweifel daran, ob der Holocaust zum Gegenstand von Fiktionen werden könne und solle (vgl. Young 1988, S. 175 f.). ${ }^{27}$ Und dieser Zweifel betrifft auch die Rezeption der Erzählung von Wander. ${ }^{28}$ Man kann nicht ohne Weiteres behaupten, dass der Text axiologisch unzuverlässig ist, wohl aber, dass seine Entscheidung, Fiktives in seiner Erzählung vom Holocaust zuzulassen, axiologische Fragen aufwirft.

\footnotetext{
${ }^{26}$ Zum Begriff der axiologischen Unzuverlässigkeit vgl. Köppe und Kindt (2014, S. 248-256). Man könnte zwar darüber streiten, ob mit dem Begriff der axiologischen und der mimetischen Unzuverlässigkeit überhaupt verwandte Phänomene bezeichnet werden. Selbst wenn sie gelegentlich gemeinsam auftreten, unterscheidet sich die Art und Weise, in der sie einmal unter dem Gesichtspunkt der Vollständigkeit und einmal unter dem der Angemessenheit vertreten werden, doch dadurch voneinander, dass das Urteil über axiologische Unzuverlässigkeit sehr viel stärker von Interpretation abhängt (vgl. Kindt 2008, S. 56) und zwar sowohl in Bezug auf die Abweichung als auch im Bezug auf den Maßstab dieser Abweichung, der sich aus den im Werk gültigen Normen ergibt. Hier werden trotzdem beide Aspekte unter dem Begriff der Unzuverlässigkeit ins Kalkül gezogen, weil sich erstens die Frage der mimetischen und der axiologischen Zuverlässigkeit des Erzählers in Bezug auf denselben Sachverhalt stellt - die Fiktionalisierung einer Holocaust-Erfahrung - und weil sich zweitens aufgrund der spezifischen Geltungsbedingungen von Holocaust-Literatur das Urteil über axiologische Zuverlässigkeit bzw. Unzuverlässigkeit mit Rücksicht auf textexterne ethische Erwartungen fällen lassen muss.

${ }^{27}$ Das Verdikt gegen die Fiktionalisierung erneuerte zuletzt Claude Lanzmann (2015).

${ }^{28}$ So mokiert sich der Rezensent der FAZ über die künstlerischen Freiheiten, die sich Wander mit seinem Material nimmt, weil er selbst den künstlerischen Umgang mit dem Material, einschließlich seiner Fiktionalisierung, für unzulässig hält: „,Wagt er sich zu weit vor, entfernt er sich zu weit vom Faktischen, so bringt ihm diese Eigenständigkeit bei aller Kunstfertigkeit kein Lob ein“ (Bächli 1985). Bächlis Rezension versteht sich aber auch als kritische Auseinandersetzung mit Christa Wolfs ausgesprochen positiver Besprechung der Erzählung Wanders. Sein Vorwurf, die Erzählung sei zu artifiziell, reagiert auf deren Behauptung, sie sei ganz kunstlos. Die textimmanente Begründung der Fiktionalität nimmt Bächli nicht zur Kenntnis.
} 
Wanders Text zeichnet sich dadurch aus, dass er kontinuierlich - nicht nur im Eingangskapitel - das Erzählen selbst zum Gegenstand macht, ${ }^{29}$ und etwa einen großen Teil der Mithäftlinge als Erzähler auftreten lässt. Erzählen ist in gewisser Weise der Gegenstand des Buches und zwar ebenso das Erzählen vom Holocaust wie das Erzählen im Holocaust. De Groot erzählt vom Leben in Amsterdam (19 ff.), Tschukran von Tours (21 f.), Meir Bernstein von seiner Familie und vom Tod seiner Tochter (44), Feinberg von den Menschen in der Rue des Rosiers in Paris (91 ff.). Bernsteins Erzählungen gleichen denen Teichmanns. Er „erzählt mit kunstvollen Ausschmückungen, beinahe so gut wie Mendel Teichmann“ (46), und aus dem Erzähler selbst ,spricht Mendel`, wie es einmal heißt. Die Erzähler sind Filiationen des einen Erzählers Mendel.

Es sind diese unüberprüfbaren Erzählungen in einer unüberprüfbaren Erzählung, die in besonderer Weise dem Verdacht der Unzuverlässigkeit ausgesetzt sind. Ihre Überprüfbarkeit wird nicht nur dem Leser, sondern auch dem Erzähler selbst entzogen. Sie handeln von einer Wirklichkeit, die der Erzähler nicht kennt und die durch niemanden sonst bezeugt wird. Insofern unterliegen auch sie jenem Beglaubigungsdefizit, das von der spezifischen epistemischen Situation des Lagers hervorgebracht wird. Vor allem zeigt sich an ihnen aber eine normative Aufladung des Erzählens als solchem, der gegenüber der historische Wahrheitsanspruch des Erzählens in den Hintergrund tritt. So zeichnet sich Wanders Text erstens dadurch aus, dass er nicht nur von Juden erzählt, sondern das Erzählen selbst jüdisch konnotiert. Alle Juden im Text sind Erzähler, während die politischen Häftlinge dezidiert keine Erzähler sind und sich eher in politischen Reden oder in Liedern artikulieren. Von einem französischen Resistant heißt es: „Seine Wortkaskaden waren nicht Poesie wie die Rede von Mendel Teichmann, sie waren die Revolution“ (80). Aus der Perspektive der Politischen stellt das Erzählen teilweise einfach eine Form jüdischer Resignation dar, während es aus der Perspektive der Juden eine Reaktion auf die Erfahrung der Diaspora ist. Meir Bernstein erzählt wie Mendel Teichmann „und andere, die seit Jahrhunderten verfolgt sind und daher im Wort leben" (46).

Mit der Figur Mendel Teichmanns, der im Text auch als „Zaddik“ (12, 30) bezeichnet wird, stellt Wander seinen Text in die Tradition chassidischen Erzählens. ${ }^{30}$ Chassidische Erzählungen sind ursprünglich mündliche Erzählungen, die von den Zaddiks, den jüdischen Weisen, handeln. Ihnen wird eine gemeinschaftsstiftende Kraft zugeschrieben. In der Regel geben sie eine Episode aus dem Leben oder einen Ausspruch des Zaddiks in kurzen, oft legendenhaften Formen wieder und vermitteln anschaulich die Lehre des Zaddiks. Allerdings

\footnotetext{
${ }^{29}$ Schmidt (2006, S. 97) spricht von der „Selbstthematisierung des Erzählens“, durch die sich Wanders Erzählung auszeichnet.

${ }^{30} \mathrm{Es}$ ist nicht ganz klar, worauf man sich bezieht, wenn man sich auf den Chassidismus bezieht. Handelt es sich um eine Erzähltradition, die für sich besteht, oder um eine heterogene Überlieferung kürzerer legendenhafter Texte, die dann eigentlich erst durch die philologische und theologische Beschreibung v. a. bei Martin Buber zu einer Erzähltradition erklärt werden, etwa
} 
unterscheidet sich der Zaddik Mendel Teichmann von denen, die man etwa in Martin Bubers zweibändiger Sammlung Die Erzählungen der Chassidim kennenlernt. Zwar teilt er mit ihnen die Bedeutung, die er für die Gemeinschaft der Juden (im Lager) hat, allerdings besteht seine Weisheit fast ausschließlich darin, dass er selbst ein Erzähler ist und dass das Exemplarische seiner Lebensführung in seiner Fähigkeit des Erzählens besteht. Das Erzählen ist nicht nur das Medium, in dem die Weisheit Teichmanns mitgeteilt wird, das Erzählen selbst ist die Weisheit Teichmanns. ${ }^{31}$ Dass schließlich der Ich-Erzähler sich für unsterblich halten kann, ist kein Ausdruck seiner Hybris, sondern die Apotheose des Erzählens selbst, die aus ihm spricht: „Die Welt nicht mehr heil. Aber ICH werde ewig leben. Ich werde glücklich sein. Werde trunken sein von Liebesgenuß“ (114). Die Glaubwürdigkeit der Apotheose besteht darin, dass sie sich mit einem spezifischen Defizit des Erzählens vom Holocaust verbindet. Alle diese Erzählungen seien schließlich, meint Ruth Klüger, euphemistisch und ,märchenhaft", weil sie notwendig von Überlebenden stammen, die gegen alle Wahrscheinlichkeit überlebt hätten. ${ }^{32}$ Wanders Ich-Erzähler begegnet dem mit seiner Anonymität. Er ist ohne Namen und ohne Geschichte, die über seine Lagergeschichte hinausginge. Er erzählt, aber nicht von sich. Die Anlage des Erzählers kann als Reaktion auf dieses Problem gelesen werden. Denn das Überleben des Erzählers wird logisch zwar vorausgesetzt, aber nicht zum Gegenstand der Erzählung gemacht. Gegenstand der Erzählung sind die, die nicht überlebt haben, und die Geschichten, die sie erzählten. Insofern macht sich der Erzähler zum bloßen Medium anderer Erzähler. ${ }^{33}$ Als solches Medium transzendiert er die konkreten Erzählungen

\footnotetext{
so, wie das Volksmärchen der Brüder Grimm eigentlich erst der philologischen Arbeit entspringt, wobei sie sich natürlich schon auf das Material der Überlieferung beziehen können. Buber selbst nimmt gelegentlich auf das Grimmsche Projekt Bezug (Buber 2015, S. 124). Wenn hier von Chassidismus die Rede ist, dann beziehe ich mich auf das, was Martin Buber damit meint. Ob man deshalb davon sprechen kann, dass Wanders Erzählung im Chassidismus „,wurzelt“ (Reiter 1995, S. 74), ist fraglich. Wander macht ja kein Geheimnis daraus, dass er „sehr wenig“ (Trampe 1997, S. 13) über das Judentum weiß. Insofern müsste man erst einmal davon sprechen, dass Wander seinen Text der Tradition des Chassidismus zuschreibt.

${ }^{31}$ In diese Richtung zielt schon Andrea Reiters Auseinandersetzung mit dem Verhältnis zwischen Chassidismus und Wanders Poetik. Allerdings lässt Reiter Wander allzu glatt aus dem Chassidismus hervorgehen. Denn es sind nicht, wie sie nahelegt, die Erzählungen selbst, die für Buber die „heilige Essenz“ wären (vgl. Reiter 1995, S. 75). Wenigstens wäre das eine sehr starke Auslegung Bubers. Vollständig heißt es bei ihm: „Aber die Erzählung ist mehr als eine Spiegelung: die heilige Essenz, die in ihr bezeugt wird, lebt in ihr fort" (Buber 2015, S. 122). Es ist also durchaus der Gegenstand oder der Inhalt der Erzählung, die für Buber als „heilige Essenz“ bezeugt wird, nicht die Erzählung selbst.

${ }^{32}$ „Die Frage, wer die Glückspilze waren [, die überlebten], führte jedoch leicht von der Statistik fort und in den Märchenwald der Erfolgsgeschichten“ (Klüger 1992, S. 106).

${ }^{33}$ Die Reduktion des subjektiven Anteils, die Tatsache, dass das Ich eben nicht einfach seine Geschichte erzählt, ist schon früh in Christa Wolfs Rezension bemerkt worden (vgl. Wolf 1972, S. 862). Debazi (2008, S. 103) erklärt sie mit der Beobachter-Position, auf die sich Wander habe zurückziehen wollen. Diese Charakterisierung ist eher zu schwach. Im Text kann man beobachten, dass sich der Erzähler eben v. a. als Erzähler der Geschichten anderer inszeniert.
} 
einzelner Häftlinge, aber auch die eigene konkrete Geschichte und verwandelt sich in ein anonymes, kollektives Subjekt, das durch kein einzelnes Individuum mehr ausgefüllt wird. Die Anonymisierung des Erzählers, die aus der Spannung zwischen der Sprecherposition (Überlebender) und seinem Gegenstand (den Toten) resultiert, wird so zur Bedingung der Möglichkeit seiner Apotheose. Sie erteilt ihm zugleich die Lizenz zur Fiktion. Denn, wie gesagt, die Anonymität eröffnet den Spielraum der Differenz zwischen Autor und Erzähler, und dass tatsächlich der Erzähler ein anderer sein möchte als sein Autor, zeigt seine Apotheose. Wäre mit dem Satz „ICH werde ewig leben“ nur der Autor oder sein Erzähler gemeint, wäre er falsch.

Dabei tut es erst einmal nichts zur Sache, dass Wanders Reanimation chassidischen Erzählens im Grunde eine Spielart einer romantischen Mythologie des Erzählens ist, die besagt, dass zeitlich und normativ mündliches Erzählen dem schriftlichen vorzuziehen sei: „Die Erzählkunst ist älter als die Literatur. [...] Im Konzentrationslager gab es keine Bücher, dort konnte sich die Kunst des Erzählens notgedrungen wieder kristallisieren. Und das habe ich in der ersten Geschichte meines Buchs mitgeteilt" (Trampe 1971, S. 7). Fast wird hier eine Sehnsucht nach Ursprünglichkeit unter den Bedingungen des Lagers eingelöst.

Und schließlich wird die Qualität der Erzählungen intradiegetisch auch gar nicht anhand ihrer historischen Wahrheit, sondern anhand ihrer Wirkung beurteilt. Mendel Teichmann findet ein „erlösendes Wort“ (14), dessen Erlösungscharakter nicht daraus resultiert, dass die Erzählung wahr ist, sondern daraus, dass sie die Verschleppten für einen Moment aus der Wirklichkeit des Lagers befreit.
Das Wort hatte magische Kräfte, es zauberte eine reichgedeckte Sabbattafel herbei, die Lieblichkeit eines jüdischen Mädchens, Duft von süßem Palästinawein und Rosinen- kuchen. Das Wort, kaum daß es erklang, machte die Männer erbleichen, es verwandelte sie, kehrte ihre Blicke nach innen, ließ sie Tränen vergießen und lachen, geißelte sie, erstickte sie, ließ sie ächzen und sogar schwitzen. (8)

Der Vergangenheitsbezug, durch den sich fast alle intradiegetischen Erzählungen auszeichnen, hat dabei im Wesentlichen die Funktion, die Personalität wieder herzustellen, die ihnen durch das Lager genommen wurde.

Er sucht die verschollenen Spuren von Schönheit in seinem Leben, sucht plötzlich einen Kumpel, der zuhören kann, und wenn er ihn gefunden hat, berauscht er sich an Vergangenem, breitet vor dem anderen Gemälde aus. Weil er es hinausschreien muß: Ich bin ein Mensch! Ich wurde geachtet! (17)

Es ist angesichts dieser enormen ethischen Aufladung des Erzählens keine Kleinigkeit, dass der Wallstein-Verlag den Text, der ursprünglich und, wie man sah, aus guten poetologischen Gründen als „Erzählung“ erschienen war, als „Roman“ wieder veröffentlicht und so mit dem verlegerischen Paratext gegen die Poetik des Textes arbeitet. ${ }^{34}$

\footnotetext{
${ }^{34}$ Wander selbst hatte Einspruch gegen die Änderung der Gattungsbezeichnung erhoben, ließ sich dann aber vom Verlag umstimmen, der seine Entscheidung mit ,verkaufstechnischen ' Gründen rechtfertigte. Persönliche Mitteilung von Susanne Wander vom 25.02.2019.
} 


\section{Unzuverlässiger Erzähler vs. Trauma}

Ich möchte mit einem kleinen Plädoyer für den unzuverlässigen Erzähler, der ja zuletzt stark in die Kritik geraten ist, ${ }^{35}$ schließen. In gewisser Weise gehört Unzuverlässigkeit zu den Kernproblemen der literaturwissenschaftlichen Forschung zur Holocaust-Literatur; freilich nicht unter dem Begriff der Unzuverlässigkeit oder der (un)reliability, sondern unter dem des Traumas. Mit diesem seit 30 Jahren in der Forschung zur Holocaust-Literatur grassierenden Begriff ${ }^{36}$ ist die Unzuverlässigkeit des Erzählens in gewisser Weise generalisiert worden. Denn er beschreibt nicht nur metaphorisch die anhaltende Beschädigung der Opfer nationalsozialistischer Gewalt, er macht auch sehr grundsätzliche Aussagen über ihre Erzählung davon. So zeichnet sich die Erzählung des/der Traumatisierten nach Lawrence Langer (2000, S. 58) durch die ,gänzliche Unfähigkeit [aus], diese Momente in irgendeiner chronologischen Ordnung zu rekonstruieren“. ${ }^{37}$ Auf der Ebene der Erfahrung hat diese Diskontinuität der Erzählung eine Entsprechung etwa in den sogenannten „Flashbacks“, die ,,auf Kosten willentlicher Erinnerung oder sogar der Kontinuität von bewußtem Denken“" gehen (Caruth 2000, S. 93). Glaubt man Slavoj Žižek, der sich der Trauma-Thetik angeschlossen hat, dann müssten wir dem zuverlässigen Erzähler einer Holocaust-Erzählung sogar in besonderer Weise misstrauen:

Wäre das Opfer in der Lage, mit klaren Worten von seiner schmerzhaften und demüitigenden Erfahrung zu berichten, eine stringente Darstellung in allen Einzelheiten zu liefern, dann würde genau das unser Mißtrauen erwecken. Der Zeuge, der zu einer klaren Schilderung seiner Erlebnisse im Lager fähig wäre, würde sich damit selbst disqualifizieren. (Žižek 2006, S. 145)

Die Unzuverlässigkeit des Erzählers fällt hier mit der Traumatisierung seines Autors zusammen. Unterschiedliche Formen der Unangemessenheit einer Erzählung können mit diesem Begriff deshalb nicht mehr beschrieben werden.

\footnotetext{
${ }^{35}$ Ohmes Einwand richtet sich wesentlich gegen die latente moralische Bewertung des Erzählers, die mit dem Begriff der „unreliability“ einhergeht (Ohme 2015, S. 134), und gründet auf der Heterogenität der erzählerischen Phänomene, die unter dem Begriff zusammengefasst werden (Ohme 2015, S. 203 ff.). Seine Vermutung ist, dass der Terminus der unreliability Phänomene unter Verdacht setzt, die man letztlich einfach als Beschreibung der Perspektive, aus der ja notwendig auch Beschränkungen resultieren, bezeichnen könnte (Ohme 2015, S. 208 f.). Versuchen, den fehlenden Maßstab für die Beurteilung von Unzuverlässigkeit, durch den Rekurs auf das ,Weltwissen' der Leser beizukommen, in Bezug auf das ein Text angemessen oder unangemessen erzählt sein kann, erteilt Ohme eine grundsätzliche Absage (Ohme 2015, S. 210-218).

${ }^{36}$ Brunner und Zajde (2011, S. 7) sprechen von einem „Paradigma“ der Holocaust-Forschung.

${ }^{37}$ Allerdings bezieht sich die Aussage bei Langer auf die mündlichen Erinnerungen. Insofern steht seine Argumentation anders als diejenige Žižeks nicht im Widerspruch zu der Tatsache, dass schriftliche Erinnerungen, literarische zumal, einem Akt der Planung entspringen, der das Werk zwar intentional nicht vollständig kontrolliert, aber doch in einem gewissen Widerspruch zur Behauptung der vollkommen unbeherrschten Erinnerung steht.
} 
Das ist nicht nur deshalb bedenklich, weil es der literaturwissenschaftlichen Analyse die Genauigkeit nimmt. Die Entdifferenzierung ist vielmehr ethisch heikel. Während man es zwar in beiden Fällen - der Erzählung des Traumatisierten und der unzuverlässigen Erzählung - mit Erzählungen zu tun hat, die auf irgendeine Art unangemessen sind, resultiert das in dem einen Fall aus der ,Unmündigkeit ‘ ihres Autors, der nicht anders kann, als deformiert zu erzählen, und in dem anderen Fall aus der Souveränität eines Autors, die es ihm erlaubt, eine bestimmte Erzählinstanz $\mathrm{zu}$ entwerfen und sich damit bestenfalls mimetisch $\mathrm{zu}-$ aus welchem Grund auch immer - beschränkten Erzählern zu verhalten (vgl. Pabst 2020).

Mit dem Begriff des Traumas geht aber auch die Möglichkeit verloren, die unterschiedlichen Motivationen und Formen zu beschreiben, mit denen Autoren Erzähler konzipieren, die nicht einfach erzählen, was der Fall ist. Das kann erstens mit betrügerischen Absichten geschehen, wie im Fall Wilkomirskis, der die Beschränkung seines Textes zwar aufdeckt, diese Beschränkung dem Leser aber als Zeichen der Authentizität ans Herz legt. Das wird möglich, weil er die Differenz zwischen Autor und Erzähler, die ja auch in diesem Text am Werk ist, verwischt. Und das Konzept des Traumas leistet dabei Vorschub. ${ }^{38}$ Das kann zweitens mit ideologischen Motiven geschehen wie im Fall von Bruno Apitz, der eine impersonale Erzählinstanz entwirft, bei der der Verdacht der Unangemessenheit der Darstellung gar keinen textimmanenten Ansatzpunkt findet. Und das kann drittens aus einer Reflexion auf die Beschränkungen resultieren, denen die Erzählung des Holocaust unterliegt, wie im Fall von Kertész Roman eines Schicksallosen oder eben bei Wander. Weil er dabei die Differenz zwischen dem Autor Wander und dem anonymen, ewigen Ich-Erzähler macht, vermeidet er, dass sein Text einfach als Wiedergabe oder als authentischer Effekt seiner Erlebnisse gelesen wird. Der Begriff, mit dem Literaturwissenschaft diese Differenz zu bezeichnen vermag, ist der des unzuverlässigen Erzählers.

Schließlich verstellt der Traumabegriff zugunsten eines vermeintlich authentischen Zugangs zum Ereignis des Holocaust dessen Beschreibung. Die Beschränkungen der Erzählung Wanders resultieren nicht einfach aus der Beschädigung des Autors, sondern aus der epistemischen Lücke und der ethischen Paradoxie, die das Ereignis des Holocaust hervorgebracht hat. Der unzuverlässige Erzähler in Wanders Text macht die Auseinandersetzung damit eigentlich erst möglich.

\footnotetext{
${ }^{38}$ Zur wechselseitigen Bestätigung des Doessecker-Textes und der Trauma-Theorie vgl. Oels (2004, S. 382-386).
} 


\section{Literatur}

Agamben, Giorgio: Was von Auschwitz bleibt. Das Archiv und der Zeuge, Frankfurt a. M. 2003.

Antelme, Robert: L'espèce humaine, Paris 2007 [1957].

Arnold, Heinz Ludwig (Hg.): Die andere Sprache. Neue DDR-Literatur der 80er Jahre [Text\&Kritik, Sonderband], in Zusammenarbeit mit Gerhard Wolf, München 1990.

Aumüller, Matthias: Offenheit und Geschlossenheit als interpretationstheoretische Funktionen des unzuverlässigen Erzählens. In: Journal of Literary Theory 12:1 (2018): Narrative Unreliability: Scope and Limits, 127-150.

Bächli, Samuel: Das Lachen der Gestiefelten. „Der siebente Brunnen“ - Eine Erzählung von Fred Wander. In: Frankfurter Allgemeine Zeitung, 17. April 1985.

Barck, Simone: Antifa-Geschichte(n). Eine literarische Spurensuche in der DDR der 1950er und 1960er Jahre, Köln/Weimar/Wien 2003.

Böttiger, Helmut: Der kleine Joschko und die Befreiung von Buchenwald. In: Süddeutsche Zeitung, 13.9.2005.

Bogdal, Klaus-Michael: Populäre Lagerliteratur. Bruno Apitz' Nackt unter Wölfen und Pierre Boulles Die Brücke am Kwai. In: Stephan Pabst (Hg.): Buchenwald in Europa, Boston/Berlin 2021 (im Erscheinen).

Brunner, José/Zajde, Nathalie: Editorial. In: dies. (Hg.): Holocaust und Trauma. Kritische Perspektiven zur Entstehung eines Paradigmas, Göttingen 2011, 7-14.

Buber, Martin: Werkausgabe, Bd. 18.1: Chassidismus III. Die Erzählungen der Chassidim, hg.v. Ran HaCohen, Gütersloh 2015.

Caruth, Cathy: Trauma als historische Erfahrung: Die Vergangenheit einholen. In: ,Niemand zeugt für den Zeugen'. Erinnerungskultur nach der Shoa, hg. v. Ulrich Baer, Frankfurt a. M. 2000, 84-98.

Debazi, Elisabeth H.: Zeugnis - Erinnerung - Verfremdung: Literarische Darstellung und Reflexion von Holocausterfahrung, Marburg 2008.

Deiss, Erika: Das Handwerk des Überlebens. In: Frankfurter Rundschau, 21.9.2005.

Faber, Elmar/Wurm, Carsten (Hg.): „Das letzte Wort hat der Minister“. Autoren- und Verlegerbriefe 1960-1969, Berlin 1994.

Graif, Gideon/Levin, Itamar: Aufstand in Auschwitz. Die Revolte des jüdischen „Sonderkommandos" am 7. Oktober 1944, Köln/Weimar/Wien 2015.

Greiser, Katrin: Sie starben keinen Opfertod. Wie das ,Kleine Lager` von Buchenwald aus der Erinnerung verschwand. In: Vielstimmiges Schweigen. Neue Studien zum DDR-Antifaschismus, hg. v. Annette Leo, Peter Reif-Spirek, Berlin 2001, 109-126.

Hähnel, Ingrid/Lemke, Elisabeth: Millionen lesen einen Roman. Bruno Apitz' „Nackt unter Wölfen“. In: Werke und Wirkungen. DDR-Literatur in der Diskussion, Leipzig 1987, 53-135.

Hantke, Susanne: Schreiben und Tilgen. Bruno Apitz und die Entstehung des BuchenwaldRomans „Nackt unter Wölfen“, Göttingen 2018.

Heymann, Stefan: Übertreibung und falsche Darstellung. In: Die Tat Nr. 45, 9.12.1945.

Kindt, Tom: Unzuverlässiges Erzählen und literarische Moderne. Eine Untersuchung der Romane von Ernst Weiß, Tübingen 2008.

Klüger, Ruth: weiter leben. Eine Jugend, Göttingen 1992.

Knigge, Volkhard (Hg.): Buchenwald. Ausgrenzung und Gewalt 1937-1945 [Ausstellungskatalog], im Auftrag der Stiftung Gedenkstätten Buchenwald und Mittelbau-Dora und in Zusammenarbeit mit Michael Löffelsender, Rikola-Gunnar Lüttgenau und Harry Stein, Göttingen 2016.

Köppe, Tilmann: Die Institution der Fiktionalität. In: Fiktionalität. Ein interdisziplinäres Handbuch, hg. v. Tobias Klauk/Tilmann Köppe, Berlin/New York 2014, 35-49.

Köppe, Tilman/Kindt, Tom: Erzähltheorie. Eine Einführung, Stuttgart 2014.

Lang, Simone Elisabeth: Unzuverlässigkeit und Heterodiegese: Überlegungen zu den Möglichkeiten und Bedingungen unzuverlässigen Erzählens in heterodiegetischen Texten. In: Journal of Literary Theorie 12:1 (2018): Narrative Unreliability: Scope and Limits, 55-76. 
Langer, Lawrence: Admitting the Holocaust, Oxford/New York 1995.

Langer, Lawrence: Die Zeit der Erinnerung. Zeitverlauf und Dauer in Zeugenaussagen von Überlebenden des Holocaust. In: ,Niemand zeugt für den Zeugen'. Erinnerungskultur nach der Shoa, hg. v. Ulrich Baer, Frankfurt a. M. 2000, 53-67.

Lanzmann, Claude: Das Unnennbare benennen. In: Frankfurter Allgemeine Zeitung, 25.1.2015.

Levi, Primo: Ist das ein Mensch?, Frankfurt a. M. 1961.

Lyotard, Jean-François: Der Widerstreit, München 1989.

McGlothlin, Erin Heather: „Das eigene Leid begreift man nicht“: Fred Wanders „Der siebente Brunnen" und die Geschichte des Selbst. In: Fred Wander. Leben und Werk, hg. v. Walter Grünzweig, Ursula Seeber, Bonn 2005, 97-118.

Meller, Marius: Der Weg ist das Paradies. In: Der Tagesspiegel, 16.3.2005.

Morgner, Irmtraud: Lob auf zwei Brote. In: Berliner Zeitung Nr. 24, 24. Januar 1973.

Niethammer, Lutz (Hg.): Der ,gesäuberte'Antifaschismus. Die SED und die roten Kapos von Buchenwald, Berlin 1994.

Niven, Bill: Das Buchenwaldkind. Wahrheit, Fiktion, Propaganda, Halle/S. 2009.

Nünning, Ansgar: Unreliable Narration. Zur Einführung: Grundzüge einer kognitivnarratologischen Theorie und Analyse unglaubwürdigen Erzählens. In: Unreliable Narration. Studien zur Theorie und Praxis unglaubwürdigen Erzählens in der englischsprachigen Erzählliteratur, hg. v. dems., Trier 1998, 3-39.

Oelke, Martina: Die Geburt der Gemeinschaft. Antisemitische Reinigungs- und Ausschlussprozesse in der DDR-Literatur am Beispiel Bruno Apitz. In: Juden. Bilder, hg. v. Heinz Ludwig Arnold, München 2008, 33-46.

Oels, David: ,A real life Grimm's fairy tale'. Korrekturen, Nachträge, Ergänzungen zum Fall Wilkomirski. In: Zeitschrift für Germanistik, N.F. Bd. 14:2 (2004), 373-390.

Ohme, Andreas: Skaz und unreliable narration. Entwurf einer neuen Typologie des Erzählers, Berlin /Boston 2015.

Pabst, Stephan: Disenfranchised Narratives. Slavoj Žižek reads Jorge Semprún. In: Holocaust Studies2020. Online DOI: https://doi.org/10.1080/17504902.2020.1769370, 1-16.

Pabst, Stephan: Kommunistische Kontrafaktur. Bruno Apitz' ,Nackt unter Wölfen“ nach Erich Maria Remarques ,Der Funke Leben“. In: Joanna Bednarska et al. (Hg.): Lagerliteratur Texte aus den Konzentrationslagern und Ghettos, Frankfurt a. M. 2021, 41-68.

Piper, Franciszek: Auschwitz, 1940-1945. Studien zur Geschichte des Konzentrations- und Vernichtungslagers Auschwitz, Bd. 3, Auschwitz 1999.

Porter, Laurence M.: Gustave Flaubert Enyclopedia, Westport 2001.

Radisch Iris: Der Herbst des Quatschocento. Immer noch, jetzt erst recht, gibt es zwei deutsche Literaturen: selbstverliebter Realismus im Westen, tragischer Realismus im Osten. In: Maulhelden und Königskinder. Zur Debatte über die deutschsprachige Gegenwartsliteratur, hg. v. Andrea Köhler/ Rainer Moritz, Leipzig 1998, 180-188.

Reiter, Andrea: „Auf dass sie entsteigen der Dunkelheit“. Die literarische Bewältigung von KZErfahrung, Wien 1995.

Ryan, Marie-Laure: The Pragmatics of Personal and Impersonal Fiction. In: Poetics 10 (1981), 517-539.

Sauter, Josef Hermann: Interviews mit Schriftstellern, Berlin 1982, 135-149.

Schmidt, Thomas: ,Unsere Geschichte'? Probleme der Holocaust-Darstellung unter DDRBedingungen: Peter Edel, Fred Wander, Jurek Becker, Teil 1 und 2. In: Monatshefte 98:1 u. 3 (2006), 83-109 und 403-426.

Stein, Harry: „Nackt unter Wölfen“ - literarische Fiktion und Realität einer KZ-Gesellschaft. In: Sehen, Verstehen, Verarbeiten. KZ Buchenwald 1937-1945, hg. v. Thüringer Institut für Lehrerfortbildung, Lehrplanentwicklung/ Medien und Stiftung Gedenkstätten Buchenwald/ Mittelbau-Dora, Bad Berka 2000, 27-40.

Thunecke, Jörg: Fred Wander's semi-autobiographical narrative, „The Seventh Well“ - „Such stories I never heard again.“. In: The Fiction of the I: Contemporary Austrian Writers and Autobiography, hg. v. Nicholas J. Meyerhofer, Riverside 1999, 242-258. 
Trampe, Wolfgang: Fred Wander „Der siebente Brunnen“ [Interview mit Fred Wander]. In: Sonntag 16.5.1971.

Trampe, Wolfgang: Gedenken, Erzählen, Leben. Gespräch mit Fred Wander. In: Argonautenschiff. Jahrbuch der Anna-Seghers-Gesellschaft 6 (1997), 13-23.

Wellm, Alfred: Morisco. Roman, Berlin/Weimar 1987.

Wander, Fred: Der siebente Brunnen. Erzählung, Berlin 1971.

Wilkomirski, Binjamin: Bruchstücke. Aus einer Kindheit 1939-1948, Frankfurt a. M. 1995.

Wolf, Christa: Gedächtnis und Gedenken. Fred Wander „Der siebente Brunnen“, Aufbau-Verlag, 1971. In: Sinn und Form 24:4 (1972), 860-866.

Young, James E.: Holocaust Documentary Fiction: The Novelist as Eyewitness. In: Writing and the Holocaust, hg. v. Berel Lang, New York/London 1988, 175-199.

Žižek, Slavoj: „Beschreibung ohne Ort“. Über den Holocaust und die Kunst/ „Description without a place“. On Holocaust and Art. In: Inaestik. Politics of Art 1 (2006), 141-161.

Zur Nieden, Susanne: „,... stärker als der Tod“: Bruno Apitz’ Roman „Nackt unter Wölfen“ und die Holocaust-Rezeption der DDR. In: Bilder des Holocaust. Literatur - Film - bildende Kunst, Köln u. a. 1997, 97-108.

Open Access Dieses Kapitel wird unter der Creative Commons Namensnennung 4.0 International Lizenz (http://creativecommons.org/licenses/by/4.0/deed.de) veröffentlicht, welche die Nutzung, Vervielfältigung, Bearbeitung, Verbreitung und Wiedergabe in jeglichem Medium und Format erlaubt, sofern Sie den/die ursprünglichen Autor(en) und die Quelle ordnungsgemäß nennen, einen Link zur Creative Commons Lizenz beifügen und angeben, ob Änderungen vorgenommen wurden.

Die in diesem Kapitel enthaltenen Bilder und sonstiges Drittmaterial unterliegen ebenfalls der genannten Creative Commons Lizenz, sofern sich aus der Abbildungslegende nichts anderes ergibt. Sofern das betreffende Material nicht unter der genannten Creative Commons Lizenz steht und die betreffende Handlung nicht nach gesetzlichen Vorschriften erlaubt ist, ist für die oben aufgeführten Weiterverwendungen des Materials die Einwilligung des jeweiligen Rechteinhabers einzuholen. 